\title{
BMJ Open 'Beyond Cancer': a study protocol of a multimodal occupational rehabilitation programme to support breast cancer survivors to return work
}

\author{
Dianne Melinda Sheppard (1) , ${ }^{1}$ Dorothy Frost, ${ }^{2}$ Michael Jefford, ${ }^{3,4,5}$ \\ Moira O'Connor, ${ }^{6}$ Georgia Halkett ${ }^{7}$
}

To cite: Sheppard DM, Frost D, Jefford $\mathrm{M}$, et al. 'Beyond Cancer': a study protocol of a multimodal occupational rehabilitation programme to support breast cancer survivors to return work. BMJ Open 2019;9:e032505. doi:10.1136/ bmjopen-2019-032505

- Prepublication history for this paper is available online. To view these files, please visit the journal online (http://dx.doi. org/10.1136/bmjopen-2019032505).

Received 24 June 2019 Revised 08 November 2019 Accepted 20 November 2019

Check for updates

(C) Author(s) (or their employer(s)) 2019. Re-use permitted under CC BY-NC. No commercial re-use. See rights and permissions. Published by BMJ.

For numbered affiliations see end of article.

Correspondence to

Dr Dianne Melinda Sheppard; dianne.sheppard@monash.edu

\section{ABSTRACT}

Introduction With more women working and surviving breast cancer, issues concerning sustainable employment must be addressed. Support to transition back to work is a gap in survivorship care. This paper describes the feasibility trial protocol for 'Beyond Cancer', a multimodal occupational rehabilitation programme to support breast cancer survivors' return to work. Breast cancer survivors are hypothesised to show improved work status, work capacity and perceived support at work at 6 months postintervention relative to baseline and a historical usual care group.

Methods and analysis The prospective feasibility design allows determination of change in primary (work status) as well as secondary outcome measures work capacity and perceived support at work. Participants: breast cancer survivors of working age, unable to work in their prediagnosis capacity for $>3$ months, their employers and a historical usual care group. Key intervention elements: an evidence-based biopsychosocial assessment and health coaching programme, employer education and support, and return to work (RTW) planning and monitoring. Health coaching empowers survivors to return to social function, including work. Employer education and support facilitates communication and improves workplace support. For employers, we predict change in confidence in effectively supporting employees' RTW. Multilevel regression modelling will provide indications of efficacy for primary and secondary outcomes, and thematic analysis will examine perceived efficacy and acceptability.

Ethics and dissemination Ethics approval has been granted by Monash and Curtin University Human Research Ethics Committees (HREC: 13300, HRE2019-0280, respectively). The evaluation of this innovative programme will provide the foundation for an Randomised Controlled Trial (RCT) and national rollout, thus improving the quality of life of those who have been directly affected by breast cancer across Australia. Results will be published in peer-reviewed journals, presented at relevant conferences and disseminated to survivorship-focused organisations.

Trial registration number Registered trial with the Australian New Zealand Clinical Trials Registry (ANZCTR) (ACTRN12618001985279); Pre-results.
Strengths and limitations of this study

- Prospective design with historical usual care comparison group enables evaluation of the implementation, acceptability and efficacy of the tailored occupational rehabilitation (OR) intervention to support the resumption of meaningful work for breast cancer survivors. The inherent limitations associated with an historical usual care comparison will be addressed by a next stage randomised controlled trial.

- Mixed-methods design using quantitative and qualitative methods to evaluate the programme.

- Addresses a recognised gap in the continuum of cancer survivorship care.

- Researchers, OR and insurance sectors, cancer support services and consumers have collaborated to design, develop resources and implement this tailored OR programme.

- Consumer involvement and input into design, training, implementation, analysis and translation.

\section{INTRODUCTION}

Breast cancer is the most common cancer in the UK, with around 54900 new breast cancer diagnoses per year accounting for $15 \%$ of all new cancer cases, ${ }^{1}$ with a similar $15.3 \%$ of new cancer diagnoses in 2018 in the USA. ${ }^{2}$ Trends indicate that in 2019, breast cancer will be the most commonly diagnosed cancer in Australia, followed by prostate cancer, colorectal cancer and melanoma. ${ }^{3}$

In Australia, 5-year relative survival rates for all cancers combined have been improving over the last 30 years, increasing from 56\% in 1986-1990 to $70 \%$ in 2011-2015 for females, ${ }^{3}$ with such gains likely due to better diagnostic methods, earlier detection and improvements in treatment. ${ }^{4}$ For breast cancer,England and Wales reported a $86.6 \%$ 5-year survival rate for 2010-2011, similar to that of Denmark, Austria and Germany. ${ }^{5}$ Australia had a slightly higher 5-year survival 
rate of $90.8 \%$ for $2011-2015 .^{3}$ Across developed nations, $\sim 40 \%-55 \%$ of cancer cases occur in people of working age (18-65 years)..$^{26}$

These statistics provide a strong case for prioritising research that addresses issues concerning sustainable employment for cancer survivors, particularly, breast cancer survivors. $^{78}$

\section{Issues for people treated for breast cancer}

For cancer survivors, common experiences that affect work ability include fatigue, pain, cognitive fluctuations, anxiety and depression, emotional adjustment issues, fear of recurrence and radiation side-effects. ${ }^{9}{ }^{10}$ As a result, increased work absence, higher risk of job change, lower income, unemployment and early retirement affect those looking to sustain or return to work (RTW) following cancer. $^{1112}$ These disease or treatment-related symptoms and associated emotional and mental health issues also impact the time taken to transition back to work at prediagnosis capacity and/or the long-term employability for cancer survivors. $^{13}$

The transition back to meaningful and sustainable work for those with breast cancer, including increasing work readiness, can be complex, especially when coupled with changing priorities such as competing family responsibilities and potential role changes. ${ }^{14} \mathrm{~A}$ supportive and flexible workplace might reduce the impact of some of these challenges, ${ }^{15} 16$ by enabling the cancer survivor to cope with reintegration into the workplace. ${ }^{17}$ While there is a positive association between higher levels of workplace support and rates of cancer survivors returning to work, ${ }^{7}$ problems at work (poor treatment, discrimination, poor accommodations) are also associated with a reduced ability to work, and with losing or leaving a job because of cancer. ${ }^{18}$ From the employer and supervisor perspective, there is lack of knowledge around how best to support breast cancer survivors through the RTW process. ${ }^{1920}$ Though generally supportive of cancer survivor employees remaining at or returning to work, supervisors may have concerns about employees being able to fulfil job demands, and are generally uncertain about how to behave around and communicate with cancer survivors. ${ }^{19-21}$ Therefore, it is often easier for an employer to send the employee off on extended sick leave to recover.

\section{The need for RTW support interventions}

Current consensus is that cancer survivors' rehabilitation should be multidisciplinary in order to address the range of (often long-term) impairments that affect daily function and quality of life, including social and work participation and financial toxicity. ${ }^{22} 23$ This can be complex because survivorship care can span a variety of specialist disciplines, each with complementary skills and expertise (eg, breast care nurse, surgeon, radiation and medical oncologists, occupational therapist, physiotherapist, exercise physiologist). The 2017 National Cancer Policy Forum Workshop highlighted that many opportunities remain to improve quality of life and outcomes for cancer survivors, particularly initiatives that facilitate returning to life, work and education that take a whole-person approach to survivorship care by addressing the psychosocial needs of the survivor as well as the physical. ${ }^{24}$ However, the major focus of follow-up treatment by cancer specialists/ oncologists tends to be managing treatment side-effects and surveillance for recurrence. ${ }^{25}$ Clinicians may not have the capacity to extend their services to provide the required support and guidance to foster the transition to work readiness. As such, this role needs to be embraced by other suitable trained professionals, such as those in the occupational rehabilitation (OR) setting.

The Australasian Faculty of Occupational and Environmental Medicine, and more recent evidence, have established that returning to appropriate work is good for the health of people with chronic conditions. ${ }^{26}$ Employees with a history of breast cancer, their employers and society are all likely to benefit from developing effective OR interventions that facilitate the return to sustainable, appropriate work. For the individual cancer survivors, sustaining work or returning to work is indicative of social recovery, regaining a sense of normality and being valued. ${ }^{1520}$ Many breast cancer survivors express the desire to RTW following, or even during, treatment. ${ }^{20}$

Within the life insurance sector, cancer is a common underlying illness relating to claims and sickness absence; it is the third most common cause of claims in Australia, ${ }^{27}$ after musculoskeletal and mental health issues, and hence, a priority. The challenge for rehabilitation professionals in tackling sickness absence and work disability generally is to work more effectively with both the individual and the employer to facilitate remaining at work or timely RTW by overcoming the barriers that are amenable to change. ${ }^{28}$ An additional challenge, particular to the life insurance context in Australia, and other systems offering similar financial disincentives, is that the vast majority of individuals receive income replacement benefits through their life insurance benefits. In addition, these individuals are often unaware that their benefits also cover 'rehabilitation expenses', which include OR services. For those who have received income replacement for lengthy periods while on sick leave, it can be difficult to see the potential benefits of participating in a 'work rehabilitation program', especially when carers, family members and clinicians tend to focus on treatment. Regardless of the system within which OR is offered, the cancer survivor typically needs to change perceptions and mindset for them to start thinking of work participation as beneficial to their return to health and wellness.

The cultural/societal perceptions about cancer are that survivors need to be supported, cared for and given time to get well. One common and misguided perception is that having time off work is best for the individual. ${ }^{26}$ The evidence behind the 'health benefits of good work' initiative strongly suggests this perception is flawed ${ }^{29}$; targeted awareness campaigns for particular populations, including cancer survivors, could prove to 
be beneficial. Finally, the sensitivity around a cancer diagnosis often means that employers, ${ }^{20}$ case managers and rehabilitation consultants are not comfortable discussing occupational support to enable individuals to sustain or resume working. This is also something that needs to be addressed. $^{19}$

\section{Cancer survivorship and work: models and frameworks}

Recent models of cancer survivorship and work suggest that both individual and workplace barriers influence work-related outcomes. ${ }^{19} 3031$ Returning to work or sustaining work for cancer survivors is complex and dependent on many factors. ${ }^{19} 32$ Breast cancer survivors can feel impaired with respect to their work ability and readiness,${ }^{20}$ and experience a variety of employment challenges.

The 'cancer and work' model ${ }^{31}$ provides a useful framework for the conceptualisation of the broad range of potential influencing factors (individual and workplace) of work outcomes for cancer survivors. Aligned with this cancer and work systems framework, ${ }^{31}$ as well as the more recent occupational therapy model for RTW for those with breast cancer, ${ }^{30}$ this tailored OR intervention will identify and modify the impact of biopsychosocial and workplace factors that could hinder transitioning to sustainable work for breast cancer survivors.

The Beyond Cancer intervention described by this protocol paper will provide individualised support through a tailored, holistic and multidimensional OR programme for women with breast cancer looking to sustain or transition back to work. This programme will be offered by a national provider of OR services, through referrals from life insurance providers and large organisations across Australia.

\section{METHODOLOGY}

\section{Trial design and outcome measures}

A mixed-methods, prospective study with historical usual care comparison group (for primary outcome comparison, that is, RTW status, work capacity) will be used to provide preliminary indications of efficacy and acceptability of the intervention, consistent with preclinical trial feasibility studies. ${ }^{33}$ Consolidated Standards of Reporting Trials guidelines will be followed for recruitment and monitoring of response rates and withdrawals. ${ }^{34}$ Primary and secondary outcome measures will be collected at baseline and at 6 months postintervention. Primary ${ }^{1}$ and secondary ${ }^{2-5}$ outcome measures include:

1. RTW status (ie, no RTW, RTW with modified hours/ duties/role, or RTW as prediagnosis)

2. Work capacity (hours, type duties)

3. Perceived support at work as measured by the Cancer Empowerment Questionnaire (CEQ) ${ }^{35}$

4. Psychosocial outcomes from the Positivum: cancer assessment.

5. Perceived effectiveness (quantitative) of the intervention. $^{36}$

\section{Study aims and objectives}

The overall study objective is to determine the feasibility of 'Beyond Cancer', a tailored, multimodal OR intervention to support the transition back to work readiness and sustainable work for breast cancer survivors. The intervention aims to change beliefs and perceptions about living with cancer and returning to work in a sample of breast cancer survivors and their employers.

\section{Detailed study aims}

1. To provide indications of intervention effectiveness, consistent with a feasibility protocol, by measuring quantifiable changes relative to a historical usual care group in primary and secondary RTW outcomes and a range of biopsychosocial secondary outcomes (see Table 1 below) from baseline to the 6 -month follow-up assessment.

2. To explore intervention acceptability by determining participants' (breast cancer survivors, their employers and trained rehabilitation consultants), perceptions of the intervention programme using postintervention interviews and focus groups.

3. To examine perceived effectiveness of the intervention as indicated by postintervention surveys, focus groups and interviews completed by employers and breast cancer survivors. In particular, survivors' perceived support during the transition back to work, as well as employers' confidence in supporting employees' RTW following breast cancer, and perceived access to, and awareness of, relevant resources to assist RTW for breast cancer survivor employees.

\section{Key hypothesis}

This feasibility study hypothesises that breast cancer survivors will show improvements at 6 months postintervention relative to baseline and a historical usual care group for the primary RTW outcome RTW status, and the secondary outcomes work capacity and perceived support at work.

\section{Recruitment and selection}

\section{Breast cancer survivors}

Prospective participants for the feasibility study are 120 breast cancer survivors aged 18-65 years (aligning with the average age of retirement in Australia of 65.5 years) unable to work in their regular (prediagnosis) capacity for at least 3 months due to breast cancer. Those eligible will be recruited over a period of 10 months nationally, across metro and regional Australia, through the following: the life insurance sector, the sickness absence claims database of recruited public and private national employers and direct referrals from cancer specialist hospitals such as Peter MacCallum in Melbourne. Recruitment for this study began on 1 May 2019.

\section{Historical usual care group}

An historical 'usual care' control cohort will be sought from the case management database from January 2015 until end December 2017. This cohort will include any 
Table 1 Data collection methods, including data source and outcome measures

\begin{tabular}{|c|c|c|c|}
\hline Data collection method / forum & $\begin{array}{l}\text { Relevant } \\
\text { aim \# }\end{array}$ & Variable(s) measured, and measurement tool & Data source \\
\hline $\begin{array}{l}\text { Tailored, evidence-based } \\
\text { assessment tool }\end{array}$ & 1 & $\begin{array}{l}\text { RTW outcomes } \\
\text { RTW status (ie, no RTW, RTW with modified } \\
\text { hours / duties / role, or RTW as pre-diagnosis). } \\
\text { Work capacity (hours, type duties). } \\
\text { Perceived support at work (CEQ). } \\
\text { Other secondary outcomes } \\
\text { Quality of life and General health items (QLQ- } \\
\text { 30). }{ }^{39} \\
\text { Pain (QLQ-30). }{ }^{39} \\
\text { Physical fatigue (BFS). }{ }^{40} \\
\text { Cognitive fatigue (BFS). }{ }^{40} \\
\text { Beliefs, perceptions, expectations of health, } \\
\text { work and employer. } \\
\text { Distress and fear of recurrence. }{ }^{42} \\
\text { Empowerment / resilience (CEQ). }{ }^{35}\end{array}$ & $\begin{array}{l}\text { Breast cancer survivors } \\
\text { (baseline, } 6 \text { months) }\end{array}$ \\
\hline $\begin{array}{l}\text { Postintervention focus groups and } \\
\text { interviews }\end{array}$ & $\begin{array}{l}3 \\
2 \\
3\end{array}$ & $\begin{array}{l}\text { Perceived effectiveness. } \\
\text { Detailed acceptability (perceptions, opinions, } \\
\text { experiences). } \\
\text { Perceptions, opinions, experiences. }\end{array}$ & $\begin{array}{l}\text { Breast cancer survivors. } \\
\text { Breast cancer survivors, } \\
\text { rehab and insurance staff. } \\
\text { Employers. }\end{array}$ \\
\hline
\end{tabular}

${ }^{*}$ The Health Education Impact Questionnaire (heiQ) is a validated 10-item instrument.

individual referred (nationally) with a diagnosis of breast cancer aged 18-65 years to OR services through IPAR Rehabilitation, the project industry partner. Initial figures indicate that this period will be adequate to obtain a cohort of similar size to the planned intervention group. It is expected that they will have similar demographics to the intervention group. The statistical comparison of the intervention and historical usual care group will control for age, time since working and time from diagnosis to referral.

\section{Procedures}

The first stage high-level inclusion screening following referral will ensure that individuals with unsuitable prognoses or circumstances are not approached to participate; this may require consultation with the breast cancer survivor's treating physician.

Subsequently, verbal consent will be sought, followed by written consent. A trained OR consultant will confirm that individuals meet the following eligibility criteria:

1. Working prior to diagnosis and treatment, but not yet RTW at full capacity

2. Ready to participate in the intervention in the context of general health/circumstances in order to build 'work-readiness'.

\section{Intervention: 'Beyond Cancer' programme}

\section{Intervention development}

In response to a need for more tailored RTW services for cancer survivors, IPAR Rehabilitation, a national provider of OR services within Australia, worked with the multidisciplinary research team to tailor the content of a holistic health coaching programme, ${ }^{37}$ already being successfully used to assist with overcoming work disability for breast cancer survivors. This programme has become known as 'Positivum: A Guide Forward after Cancer ${ }^{\mathrm{TM}}$ '. A unique stakeholder collaboration including representatives from the OR sector, life insurance and cancer support sectors, and research academics, developed the tailored biopsychosocial assessment and health coaching content for cancer survivors looking to sustain or RTW. The involvement of a cancer support sector employee ensured sensitivity to the emotional and physical stressors prevalent during and following diagnosis and treatment for cancer.

A key feature of the Beyond Cancer OR intervention is the Positivum health coaching sessions which are flexibly delivered predominantly face-to-face to suit individual needs and circumstances. Self-management support, including health coaching, is commonly and effectively used within the chronic disease rehabilitation setting to develop a broad set of attitudes, behaviours and skills to better manage the impact of chronic conditions on quality of life. ${ }^{28}$ In this context, health coaching principles have the potential to minimise the negative impact of individual beliefs and expectations on RTW, through empowering and improving self-efficacy. ${ }^{14}$

Research suggests that to effectively address workplace/ employer challenges encountered by breast cancer survivors seeking to remain at or RTW, interventions should include a workplace component. ${ }^{38}$ To address potential workplace barriers, therefore, the 'Beyond Cancer' intervention will also offer an evidence and expert stakeholder-informed workplace component. Taken 


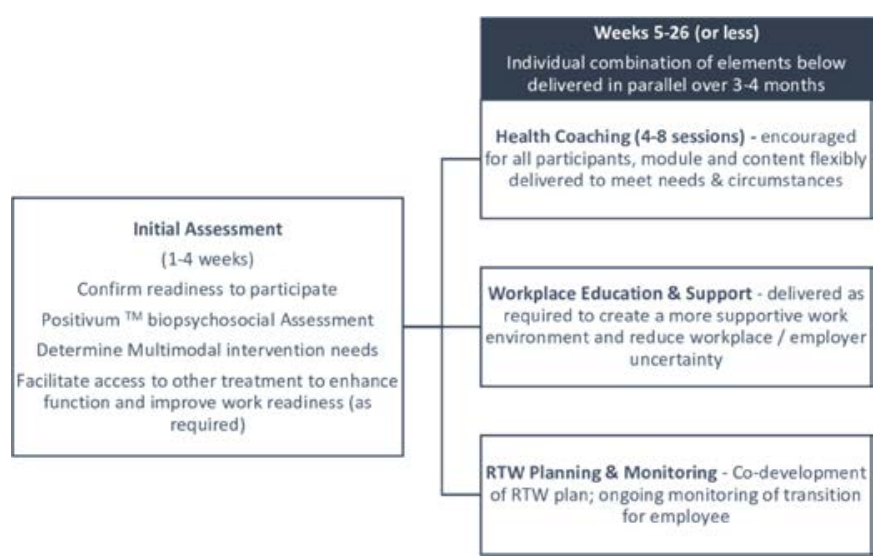

Figure 1 Schematic illustration demonstrating tailored Beyond Cancer intervention delivery.

into consideration in particular were recent qualitative research findings, recommending:

1. Education for the employer about cancer survivors' needs and how best to support their transition back to work.

2. Consideration of the timing of the intervention-early stages postdiagnosis is best.

3. Providing resources to aid communication between the supervisor/employer and the cancer survivor (for both parties).

4. The promotion of a 'shared responsibility' for RTW between the cancer survivor and the employer.

5. Training for rehabilitation professionals about cancer survivorship and work-readiness needs. ${ }^{18} 2130$

\section{Beyond Cancer programme: overview of content and delivery framework}

The Beyond Cancer programme will be delivered in a tailored fashion using predominantly face-to-face OR consultation sessions, as required, by a trained OR consultant. The intervention has three main components, the delivery and doses of which are determined by the experienced, trained OR consultant and the results of the biopsychosocial Positivum assessment which identifies key barriers and facilitators to work readiness (see figure 1).

The online assessment is delivered during the first session with the OR consultant (within the first 4 weeks of referral). The assessment results are subsequently used to inform and tailor the subsequent services and support provided as part of the Beyond Cancer intervention (see below).

The tailored intervention elements available to breast cancer survivors are to be delivered in parallel, and as required, from around week 5 after referral into the programme. In cases where the OR consultant and participant decide that more than one intervention element would be useful, subsequent sessions would include time spent on each of these elements in order to progress and build work readiness for that individual. Delivery of the Beyond Cancer programme will vary by virtue of it being tailored to the individual; however, based on a pilot of the programme, it is expected to take a minimum of 12 and a maximum of 26 weeks. The health and well-being needs and circumstances of each breast cancer survivor also influences the programme duration.

\section{Procedure: first meeting and initial assessment}

The initial assessment will be conducted by their trained OR consultant who will remain consistent throughout the programme. During this initial face-to-face meeting, breast cancer survivors will provide consent to participate, and complete the online Positivum biopsychosocial assessment tool which takes $\sim 15 \mathrm{~min}$ (see below). Consent to liaise with the employer will also be sought during this initial or subsequent meetings, as appropriate. Ultimately, and at a comfortable and appropriate pace, the primary goal of the flexible participation in the Beyond Cancer intervention is to facilitate work readiness and the gradual return to sustainable, suitable work. This is achieved through a partnership between the OR consultant and the breast cancer survivor, and in consultation with the primary physician, other health practitioners (eg, exercise physiologists) and the employer as required (Expert panel recommendations were NOT to mandate employer involvement, if it was considered unnecessary, and if pursued, obtaining employee (cancer survivor) consent to involve the employer is essential). Where required, the OR consultant will liaise with other treatment professionals (eg, exercise physiologists trained in cancer survivorship) to enhance function and work readiness.

\section{Positivum: cancer assessment}

An expert panel consisting of two researchers with expertise in work-related disability and RTW, a senior OR representative and a cancer support service consultant developed, trialled and refined the Positivum: cancer assessment tool. The factors were informed by the current literature on psychosocial determinants of RTW for cancer survivors generally. The Positivum assessment, delivered online and implemented at baseline and 6 months postintervention, consists of 50 items with Likert-style responses. Items within each factor are predominantly drawn from a mixture of several validated instruments (see table 1 and descriptions). The scoring of each of the validated instruments is adhered to, but for ease of interpretation, is transformed into an individual factor score out of 100. Participants, therefore, receive a score (relative to $100 \%$ 'best possible' function) for a range of psychosocial factors, entailing:

1. Quality of life and general health: these two single standalone items are from the QLQC-30, ${ }^{39}$ a quality of life instrument developed by the European Organisation for Research and Treatment of Cancer, and have been validated for use with cancer populations. These items rate perceptions of breast cancer survivor's overall health and quality of life on a Likert scale ranging from 'very poor' to 'excellent'. The maximum possible score of 100 is obtained when both items receive the response excellent. 
2. Pain: also from the QLQC-30, ${ }^{39}$ these two items measure the presence of pain and the extent of interference with daily activities on a scale from 'Not at all' to 'Very much'. The maximum possible score of 100 is obtained when both items receive the response very much.

3. Physical fatigue: from the Bidimensional Fatigue Scale (BFS),${ }^{40}$ these eight items are a validated subfactor of the BFS, and capture the extent of physical fatigue by measuring the presence of symptoms such as 'tiredness', 'lacking in energy' 'weakness', on a scale from 'better than usual' to 'much worse than usual'. The maximum possible score of 100 is obtained when all eight items receive the response better than usual.

4. Cognitive fatigue: also from the $\mathrm{BFS},{ }^{40}$ these six items represent the second validated subfactor that captures the extent of cognitive fatigue. These items measure the presence of symptoms such as 'difficulties thinking clearly', concentrating, or 'slips of the tongue' on a scale from better than usual to much worse than usual. The maximum possible score of 100 is obtained when all six items receive the response better than usual.

5. Beliefs, perceptions and expectations of health, work and employer: these 15 items were internally developed (no validated scales were identified in the literature that captured these concepts) based on consistent evidence that an individual's beliefs, perceptions and expectations influence work and health outcomes in the OR setting. Items were developed by an expert panel (see below) to assess an individual's beliefs and perceptions relating to their health, current function and work, as well as expectations for recovery and commencement of work. Feedback from end-users (clients and OR consultants) as well as initial results showing improvements following OR service provision have allowed us to establish face validity for the beliefs and perceptions construct. Further, repeat initial assessment results from a sample of 30 individuals will allow us to examine test-retest reliability of these items. The maximum possible score of 100 is obtained for each subfactor when all items receive the response reflecting the most positive beliefs and perceptions ('strongly disagree' for 11 items and 'strongly agree' for four items).

6. Distress and fear of recurrence: this factor was designed to capture the presence of psychological distress specific to cancer survivors. It comprises five items including the Distress Thermometer (single item), ${ }^{41}$ and the Concerns about Recurrence Questionnaire (CARQ4). ${ }^{42}$ The former is a measurement of the distress experienced in the past week on a scale of $0=$ no distress to $10=$ extreme distress, and the latter four items the presence of 'worry' about, for example, diagnostic tests, cancer returning, health (five-point Likert scale from 'none of the time' to 'all of the time'). The maximum possible score of 100 is obtained when the distress thermometer indicated 'no distress' and all four CAR-Q items receive the response none of the time.
7. Empowerment / resilience: eight of 19 items of the 'Personal Strength' subscale of the $\mathrm{CEQ}^{35}$ that capture the construct of psychological empowerment for the cancer survivor. Questions assess life satisfaction and purpose, perceived self-worth and general self-efficacy. Each item is measured on a five-point Likert Scale from strongly disagree to strongly agree. The maximum possible score of 100 is obtained when all eight items receive the response strongly agree.

8. Perceived support at work: four of the six items of the 'Work' subscale of the CEQ ${ }^{35}$ that assess the extent to which the individual feels supported at work. Each item is again measured on a five-point Likert Scale from strongly disagree to strongly agree. The maximum possible score of 100 is obtained when all four items receive the response strongly agree.

The assessment results are presented as a high-level individual profile showing each of the above factor scores in a standardised report format that is easily interpreted by the trained OR consultant and the cancer survivor.

Consultant guidelines informed by an expert panel guide the flexible implementation of the Beyond Cancer intervention, and ensure that the most relevant elements of the intervention and the most relevant content or services from each element are delivered. The tailoring is an ongoing process throughout the 12-week to 26-week delivery of the Beyond Cancer programme in order to meet the changing rehabilitation needs and circumstances of the cancer survivor. In addition, the tailoring of the Beyond Cancer intervention is jointly decided by the trained, experienced OR consultant and the cancer survivor; the OR consultants have received cancer survivor-specific training, and are suitably qualified and experienced.

The Positivum assessment results identify individuals' strengths and weaknesses across a range of biopsychosocial constructs (see above, under 'Positivum: Cancer assessment'). To illustrate the tailoring of the intervention content, cancer survivors who score low on 'perceived support at work' would be encouraged by their consultant to discuss and problem-solve around the potential lack of support within the workplace. The discussion about the Positivum profile results would take into consideration any other barriers identified, such as physical or mental fatigue, and/or high levels of psychological distress. The presence of any key support factors, such as social support, or support in the workplace, are also identified, and the consultant is then in a position to have informed discussions with the cancer survivor about the most relevant first steps in the Beyond Cancer programme (see Procedure subsections below for details of each of the programme components). These early sessions are critical in establishing the level of need for intervention support and services, as well as the type of services (intervention components) that will likely be required to best support the individual with moving towards work readiness. On occasion, the initial Positivum assessment results and follow-up discussions between the consultant 
and survivor will indicate currently overwhelming circumstances and barriers such that any discussion about work readiness is likely to cause distress. In these situations, discussions about transitioning back to work are deferred, and the consultant would instead focus on ensuring that these individuals are provided with additional supports, resources and services required to assist with their current issues.

Procedure: health coaching tailored to the breast cancer survivor The Positivum: A guide forward after cancer health coaching programme was designed to reduce the impact of any existing negative beliefs and perceptions about work and health, and other biopsychosocial factors that hinder work readiness for cancer survivors. Participation in the Positivum health coaching is encouraged for all participants as the modules can be tailored to the specific needs of the individuals. The module content theoretically aligns with the cancer and work model ${ }^{31}$; a useful framework within which to consider the broad range of potential facilitators and barriers (individual and workplace) to optimal work outcomes for breast cancer survivors. Principles of behaviour change theory, ${ }^{43}$ are also used throughout in the context of facilitating more positive and helpful beliefs and perceptions about health and work. ${ }^{37}$ The content assists with the early stages of thinking about how to approach RTW, building work readiness and RTW planning. The information, content and resources are current, evidence-based and have been informed by experts in cancer survivorship, including end-users. Seven tailored health coaching modules build health literacy and self-management skills and cover, for example, problem solving around managing breast cancer symptoms and treatment side-effects (eg, physical and cognitive fatigue, fear of recurrence) in the workplace, and the importance of healthy lifestyle factors such as graded exercise, sleep and social support from family / friends. Also covered is the evidence about the health benefits of work in the context of being a cancer survivor, ${ }^{20}$ and a module on building resilience.

The health coaching sessions are offered flexibly, and typically delivered across 8-12 weeks with a single 1 hour session every 1-2 weeks. They are delivered individually, and ideally, face-to-face with some 'take home' exercises within the manual completed in their own time. Importantly, the content is delivered in a guided fashion (ie, in partnership with the OR consultant), to suit the circumstances of the individual breast cancer survivor.

\section{Procedure: workplace / employer education and support}

Where appropriate, and with consent from the survivor, the consultant will liaise with the employer to clarify how the programme could support the transition back to sustainable work. This intervention component is also tailored to issues affecting women with breast cancer. It aims to create a more supportive work environment by educating the employer about the unique needs of breast cancer survivors and reducing employer and coworker uncertainty around aspects of diagnosis and rehabilitation. Early initial discussions with the workplace / employer are encouraged to establish a line of communication. Trained OR consultants then liaise with an appropriate workplace representative (eg, manager, HR representative) to provide education and resources to employers, including:

1. How to approach discussions with the employee about their plans to remain at work or transition back to work.

2. Considering 'reasonable adjustments' to work role(s) and hours that may assist with making the transition to work as comfortable as possible for all concerned.

3. The importance of seeking advice from the employee about their unique needs and wishes in regards to disclosing health information.

4. Preparing other staff for the return of the employee, including role adjustments, changes in appearance, work capacity (eg, fatigue, ability to concentrate).

Note that although employers have legal obligations to support employees resume work after cancer, the OR consultants will not compel them to participate.

\section{Procedure: RTW planning and monitoring}

RTW planning and monitoring will be tailored to individual circumstances by the trained OR consultant, and will ideally begin before work readiness has been fully established (in order to align with best practice OR principles or early intervention). Making a judgement regarding an individual's work readiness is a core skill of OR consultants. As perceived work readiness may not reflect an individual's actual ability to return to suitable, modified duties / hours, it is important for the consultant to use their well-practiced judgement in this regard. A graded RTW plan developed in consultation with the survivor, employer and primary physician typically starts slowly, is monitored and builds up to ensure a (gradual) return to sustainable work and quality of life that meets the needs of the breast cancer survivor employee. An initial review of duties explores the demands of the workplace and the unique needs (ie, current health status and physical, emotional and cognitive capacity) of the employee. If no longer employed, these services may include preparing for a new job (job seeking services). The process will also take into consideration the potential for lack of insight; the breast cancer survivor may not realise how challenging / tiring the RTW process can be until she returns to the workplace (eg, even getting to and from work may be tiring).

\section{Patient and public involvement}

Patient and members of the public will be involved at several stages of the study, including the intervention design, implementation and evaluation. We received input from cancer survivors with experience of RTW following diagnosis, as well as cancer support service employees at the design and planning stages as part of an expert panel and will also seek similar input when refining the focus 
group and interview questions at the evaluation stage. The development of OR consultant training workshop and materials has also drawn on the recommendations of the assembled expert panel including the research team, ${ }^{44}$ cancer support services personnel (Cancer Council NSW), a medical oncologist, a consumer, an occupational physician, an employer representative and an OR consultant with experience in providing similar support and services to cancer survivors. We intend to disseminate the summary results to trial participants and will seek assistance from the cancer support service organisations to ensure a broad reach.

\section{Sample size and power calculation}

As this is a novel multimodal intervention with unknown effect sizes for our primary and secondary outcome measures, this feasibility study will establish effect size to enable a more accurate determination of the required sample size for the next phase randomised controlled trial. The estimation of sample size for the purpose of the feasibility trial was based on a Cochrane review paper that examined the evidence for enhanced RTW as a result of work support interventions in cancer survivors. de Boer et $a l^{11}$ reported moderate quality evidence for multidisciplinary interventions (best fit to this protocol) leading to higher RTW rates over 'care as usual'. The relative risk reported was 1.15 (95\% CI 1.01 to 1.30$).{ }^{11}$ Moreover, past research has indicated that, on average, $\sim 60 \%$ cancer survivors RTW after 12 months, ${ }^{11}$ and that there is a steady increase in RTW rate from 6 to 24 months postdiagnosis. ${ }^{45}$ Given an estimated average length of time since diagnosis of 18 months (based on the pilot sample recruited from the life insurance sector), a usual care RTW incidence of 0.80 was used for the calculations. Based on EpiTools epidemiological software, with a power of $80 \%$ and twosided significance level of $\mathrm{p}<0.05$, the sample size for a full cohort study was estimated to be 128 cancer survivors in each arm. For this feasibility protocol, therefore, sample size was set just below this figure at $n=120$.

\section{Data management and analysis}

The prospective design and comparison of baseline and 6-month follow-up data will enable identification of changes in primary and secondary outcome measures. The use of a historical usual care group will enable the identification of any changes in work status and work capacity over a similar period of time in a comparable cancer survivor group not receiving the intervention. Quantitative analyses including multilevel regression modelling will be applied to examine the effect of the intervention on primary and secondary outcome measures. Additional exploratory analyses, consistent with feasibility study designs, such as binary logistic regression and correlations will be used to explore whether intervention components are predictors of key outcome variables. Further, multilevel regression modelling will provide preliminary evidence of the effect of the intervention on the biopsychosocial secondary outcomes as measured by the Positivum assessment. The authors note that the latter analyses will lack the usual care comparison, and are likely to be underpowered based on the number of secondary outcome variables. They will, however, provide useful indications of effect size to determine required sample size for the next stage RCT.

For the qualitative data, a systematic, inductive thematic analysis approach aligned with the focus group data analysis framework, ${ }^{46}$ will be used to analyse the data from focus groups and interviews with breast cancer survivors and their employers. A standardised thematic analysis with six phases, ${ }^{47}$ will be performed separately by two researchers, culminating in a final discussion and refining of themes until consensus is reached.

\section{Rehabilitation consultant expertise and training}

The OR consultants are qualified allied health professionals (most commonly occupational therapists, psychologists and exercise physiologists). When selecting consultants for the role in the Beyond Cancer programme, an experienced OR manager considers the suitability of each consultant against a set of competencies / inherent characteristics, such as empathy, respect, genuineness that help to build rapport, engagement and trust.

In addition, all consultants will receive specialised breast cancer survivor training prior to receiving referrals into the Beyond Cancer intervention. The training consists of a 4-hour workshop that features:

1. A presentation delivered by an experienced and senior employee from a cancer support service (not-forprofit) organisation, Cancer Council NSW that covers cancer survivorship, unique needs of breast cancer survivors and resources available.

2. A presentation from a breast cancer survivor about personal experiences during their transition back to work.

3. An introduction to the Beyond Cancer intervention, the research project and the importance of recording key outcomes within the database.

4. An interactive role play showcasing how best to have initial key conversations with the breast cancer survivor.

5. A training segment to refresh consultants' skills on how to deliver the Positivum assessment and health coaching.

\section{DISCUSSION}

The Beyond Cancer OR intervention is unique in the way that it brings together the life insurance, OR and cancer support sectors to benefit breast cancer survivors looking to transition back to work and their employers. This feasibility study will implement and evaluate 'Beyond Cancer', a rehabilitation programme to support breast cancer survivors to return to work readiness with a sample of 120 breast cancer survivors.

In the short term, this study will implement the unique OR intervention using an individualised, tailored service 
delivery model for breast cancer survivors. Initial stages will upskill and train OR consultants in breast cancer survivor-specific support, especially in the area of communication and unique needs. During the tailored delivery, as appropriate, the intervention will alsoprovide relevant resources and education to a variety of workplaces to enable more effective practices when supporting continued employment / transition back to work for breast cancer survivors.

The feasibility study will provide results that will allow the research team to determine the required sample size and demonstrate recruitment feasibility in the lead up to a nation-wide randomised controlled trial, or largeorganisation supported roll-out. It will also provide the research team with recommendations to refine the intervention and/or its implementation.

In the long term, this study will contribute to a better understanding of the individual factors and characteristics that contribute to the successful, staged return to meaningful and sustainable work for breast cancer survivors, thus contributing to cancer and work models. Moreover, the model currently being evaluated could be readily implemented within other commonly occurring cancers in people of working age, such as head and neck cancers and melanomas, following appropriate consultation with experts in these cancer fields and tailoring programme content accordingly.

The results of the feasibility study will be disseminated broadly through publication in scientific journals, national and international conference presentations, media releases, national cancer support agencies, stakeholder reports and presentations.

\section{CONCLUSION}

This intervention represents an innovation in breast cancer care that addresses a gap by acknowledging work as an important part of rehabilitation for women with breast cancer. This project works towards improved work and quality of life outcomes for breast cancer survivors, as well as improvements in practices and service offerings within life insurance, OR and large employer sectors. If broadly implemented at the national level, this OR intervention tailored to breast cancer survivors has the potential to improve the work-related and quality of life outcomes of breast cancer survivors, their families and society.

\footnotetext{
Author affiliations

${ }^{1}$ Monash University Accident Research Centre, Monash University, Clayton, Victoria, Australia

${ }^{2}$ Research and Innovation, MedHealth Group, Melbourne, Victoria, Australia ${ }^{3}$ Department of Cancer Experiences Research, Peter MacCallum Cancer Centre, Melbourne, Victoria, Australia

${ }^{4}$ Sir Peter MacCallum Department of Oncology, The University of Melbourne, Parkville, Melbourne, Victoria, Australia

${ }^{5}$ Australian Cancer Survivorship Centre, a Richard Pratt legacy, Peter MacCallum Cancer Centre, Melbourne, Victoria, Australia

${ }^{6}$ School of Psychology, Curtin University Bentley Campus, Perth, Western Australia, Australia
}

${ }^{7}$ School of Nursing, Midwifery and Paramedicine, Curtin University Bentley Campus, Perth, Western Australia, Australia

Contributors DMS: principle writing role for the protocol paper and funding application. DF: industry collaborator / research partner and expert advisor, reviewed and helped refine protocol paper prior to submission, and contributed many hours to the funding application. MJ: clinical (medical oncology) collaborator and expert advisor, reviewed and helped refine protocol paper prior to submission, and contributed many hours to the funding application. M0: senior academic (psychology, cancer survivorship) collaborator and expert advisor, reviewed and helped refine protocol paper prior to submission, and contributed many hours to the funding application as a chief investigator. GH: senior academic (cancer survivorship and supportive care) partner and expert advisor, reviewed and helped refine protocol paper prior to submission, and contributed many hours to the funding application as primary chief investigator.

Funding This work was supported by the National Breast Cancer Foundation, grant number IIRS-18-071. The pilot was supported by AIA Australia. SwissRe and IPAR Rehabilitation are key industry partners.

Competing interests None declared.

Patient consent for publication Not required.

Ethics approval Monash University Human Research and Ethics Committee: 13300; Curtin University Human Research Ethics Committee: HRE2019-0280.

Provenance and peer review Not commissioned; externally peer reviewed.

Open access This is an open access article distributed in accordance with the Creative Commons Attribution Non Commercial (CC BY-NC 4.0) license, which permits others to distribute, remix, adapt, build upon this work non-commercially, and license their derivative works on different terms, provided the original work is properly cited, appropriate credit is given, any changes made indicated, and the use is non-commercial. See: http://creativecommons.org/licenses/by-nc/4.0/.

ORCID iD

Dianne Melinda Sheppard http://orcid.org/0000-0002-9928-1740

\section{REFERENCES}

1 Cancer research UK. Available: https://www.cancerresearchuk. org/health-professional/cancer-statistics/statistics-by-cancer-type/ breast-cancer/survival\#heading-Zero [Accessed April, 2019].

2 National Cancer Institute. Surveillance, epidemiology and end results (SEER) program: National Institutes of health, 2019. Available: https://seer.cancer.gov/ [Accessed June 2019].

3 Australian Institute of Health and Welfare (AlHW). Cancer in Australia 2019 (report No.: can 123. Canberra: AlHW, 2019.

4 Dickman PW, Adami HO. Interpreting trends in cancer patient survival. J Intern Med 2006;260:103-17.

5 De Angelis R, Sant M, Coleman MP, et al. Cancer survival in Europe 1999-2007 by country and age: results of EUROCARE--5-a population-based study. Lancet Oncol 2014;15:23-34.

6 Cancer Council NSW. $7.7 \mathrm{~m}$ days lost to prostate and breast cancer by 2022, 2015. Available: http://www.cancercouncil.com.au/60513/ news-media/latest-news-news-media/media-releases-news-roomnews-media/7-7m-work-days-lost-to-prostate-and-breast-cancerby-2022/ [Accessed Dec 2018].

7 Cancer Council Australia. Working with cancer: a workplace resource for leaders, managers, trainers and employees, 2006. Available: http://www.cancer.org.au/content/pdf/AboutCancer/support/ workingwithcancer_intro.pdf\#_ga=1.232497247.1101651681. 1453956222

8 Australian Institute of Health and Welfare (AlHW). Cancer in Australia 2017 (contract No.: cat. No. can 100. Canberra: AlHW, 2017.

9 Taskila T, de Boer AGEM, van Dijk FJH, et al. Fatigue and its correlates in cancer patients who had returned to work--a cohort study. Psychooncology 2011;20:1236-41.

10 Tiedtke C, de Rijk A, Dierckx de Casterlé B, et al. Experiences and concerns about 'returning to work' for women breast cancer survivors: a literature review. Psychooncology 2010;19:677-83.

11 de Boer AG, Taskila T, Tamminga SJ, et al. Interventions to enhance return-to-work for cancer patients. Cochrane Database Syst Rev 2011:CD007569.

12 Mehnert A, de Boer A, Feuerstein M. Employment challenges for cancer survivors. Cancer 2013;119 Suppl 11:2151-9.

13 Munir F, Yarker J, McDermott H. Employment and the common cancers: correlates of work ability during or following cancer treatment. Occup Med 2009;59:381-9. 
14 Cimprich B, Janz NK, Northouse L, et al. Taking charge: a selfmanagement program for women following breast cancer treatment. Psychooncology 2005;14:704-17.

15 Kennedy F, Haslam C, Munir F, et al. Returning to work following cancer: a qualitative exploratory study into the experience of returning to work following cancer. Eur J Cancer Care 2007;16:17-25.

16 Tiedtke C, Dierckx de Casterlé B, Donceel P, et al. Workplace support after breast cancer treatment: recognition of vulnerability. Disabil Rehabil 2015;37:1770-6.

17 Duijts SFA, van Egmond MP, Gits M, et al. Cancer survivors' perspectives and experiences regarding behavioral determinants of return to work and continuation of work. Disabil Rehabil 2017;39:2164-72.

18 Moskowitz MC, Todd BL, Chen R, et al. Function and friction at work: a multidimensional analysis of work outcomes in cancer survivors. $J$ Cancer Surviv 2014;8:173-82.

19 Fitch MI, Nicoll I. Returning to work after cancer: survivors', caregivers', and employers' perspectives. Psychooncology 2019;28:792-8.

20 Tiedtke C, Donceel P, de Rijk A, et al. Return to work following breast cancer treatment: the employers' side. J Occup Rehabil 2014;24:399-409.

21 Yagil D, Goldblatt H, Cohen M. Dyadic resources in the return to work of cancer survivors: exploring supervisor-employee perspectives. Disabil Rehabil 2019;41:2151-8.

22 Pearce A, Tomalin B, Kaambwa B, et al. Financial toxicity is more than costs of care: the relationship between employment and financial toxicity in long-term cancer survivors. J Cancer Surviv 2019;13:10-20.

23 Silver JK, Baima J, Newman R, et al. Cancer rehabilitation may improve function in survivors and decrease the economic burden of cancer to individuals and society. Work 2013;46:455-72.

24 Kline RM, Arora NK, Bradley CJ, et al. Long-Term Survivorship Care After Cancer Treatment - Summary of a 2017 National Cancer Policy Forum Workshop. J Natl Cancer Inst 2018;110:1300-10.

25 Jefford M, Ward AC, Lisy K, et al. Patient-reported outcomes in cancer survivors: a population-wide cross-sectional study. Support Care Cancer 2017;25:3171-9.

26 The Royal Australasian College of Physicians. What is good work? Position statement \& Companion statement to 'Realising the health benefits of work. Australasian Faculty of Occupational and Environmental Medicine, 2013.

27 SwissRe. Rehabilitation Watch 2016. Sydney, Australia, 2017.

28 Ellis N, Sheppard DM, Battersby MW. A review of self-management support for (work-related) musculoskeletal pain disorders. In: Gatchel RJ, Schultz IZ, eds. The Handbook of musculoskeletal pain and disability disorders in the workplace. handbooks in health, work, and disability. New York: Springer Science+Business Media, 2014: 395-416.

29 The Royal Australasian College of Physicians. Realising the health benefits of work - an evidence update. The Australasian Faculty of Occupational \& Environmental Medicine, 2015.

30 Désiron HAM, Crutzen R, Godderis L, et al. Bridging health care and the workplace: formulation of a return-to-work intervention for breast cancer patients using an intervention mapping approach. J Occup Rehabil 2016;26:350-65.
31 Feuerstein M, Todd BL, Moskowitz MC, et al. Work in cancer survivors: a model for practice and research. $J$ Cancer Surviv 2010;4:415-37.

32 Dewa CS, Trojanowski L, Tamminga SJ, et al. Work-related experiences of head and neck cancer survivors: an exploratory and descriptive qualitative study. Disabil Rehabil 2018;40:1252-8.

33 Bowen DJ, Kreuter M, Spring B, et al. How we design feasibility studies. Am J Prev Med 2009;36:452-7.

34 Schulz KF, Altman DG, Moher D, et al. Consort 2010 statement: updated guidelines for reporting parallel group randomised trials. BMC Med 2010;8:18.

35 van den Berg SW, van Amstel FKP, Ottevanger PB, et al. The cancer empowerment questionnaire: psychological empowerment in breast cancer survivors. J Psychosoc Oncol 2013;31:565-83.

36 Osborne RH, Elsworth GR, Whitfield K. The health education impact questionnaire (heiQ): an outcomes and evaluation measure for patient education and self-management interventions for people with chronic conditions. Patient Educ Couns 2007;66:192-201.

37 Sheppard DM, Frost D. Beliefs and perceptions in relation to health and work. International Conference of Behavioural Medicine; 9th December, 2016, Melbourne, Australia, 2016.

38 Cullen KL, Irvin E, Collie A, et al. Effectiveness of workplace interventions in return-to-work for musculoskeletal, pain-related and mental health conditions: an update of the evidence and messages for practitioners. J Occup Rehabil 2018;28:1-15.

39 Aaronson NK, Ahmedzai S, Bergman B, et al. The European organization for research and treatment of cancer QLQ-C30: a quality-of-life instrument for use in international clinical trials in oncology. J Natl Cancer Inst 1993;85:365-76.

40 Chalder T, Berelowitz G, Pawlikowska T, et al. Development of a fatigue scale. J Psychosom Res 1993;37:147-53.

41 Gessler S, Low J, Daniells E, et al. Screening for distress in cancer patients: is the distress thermometer a valid measure in the UK and does it measure change over time? A prospective validation study. Psychooncology 2008;17:538-47.

42 Thewes B, Zachariae R, Christensen S, et al. The concerns about recurrence questionnaire: validation of a brief measure of fear of cancer recurrence amongst Danish and Australian breast cancer survivors. J Cancer Surviv 2015;9:68-79.

43 Heymans MW, de Vet HCW, Knol DL, et al. Workers' beliefs and expectations affect return to work over 12 months. J Occup Rehabil 2006;16:685-95.

44 Halkett G, O'Connor M, Aranda S, et al. Communication skills training for radiation therapists: preparing patients for radiation therapy. J Med Radiat Sci 2016;63:232-41.

45 Mehnert A, Koch U. Predictors of employment among cancer survivors after medical rehabilitation - a prospective study. Scand $J$ Work Environ Health 2013;39:76-87.

46 Nili A, Tate $M$, Johnstone $D$, et al. A framework and approach for analysis of focus group data in information systems research. Communications of the Association for Information Systems 2017;40:1-21.

47 Braun V, Clarke V. Using thematic analysis in psychology. Qual Res Psychol 2006;3:77-101. 